\title{
Self-Similar Tiling Systems, Topological Factors and Stretching Factors
}

\author{
María Isabel Cortez • Fabien Durand
}

Received: 15 June 2006 / Revised: 28 August 2007 / Accepted: 30 August 2007 /

Published online: 2 October 2008

(C) Springer Science+Business Media, LLC 2008

\begin{abstract}
In this paper we prove that if two self-similar tiling systems, with respective stretching factors $\lambda_{1}$ and $\lambda_{2}$, have a common factor which is a nonperiodic tiling system, then $\lambda_{1}$ and $\lambda_{2}$ are multiplicatively dependent.
\end{abstract}

Keywords Tiling $\cdot$ Self-similar $\cdot$ Factor maps $\cdot$ Stretching factor $\cdot$ Cobham theorem

\section{Introduction}

Given a nonperiodic self-similar tiling $\mathcal{T}$ generated by some similarity $S_{1}$ with stretching factor $\lambda_{1}$, it is rather natural to ask if we could generate $\mathcal{T}$ using another similarity with a different stretching factor $\lambda_{2}$. This is of course possible taking a power of the similarity $S_{1}$, where $\lambda_{2}$ is in this case a power of $\lambda_{1}$. Holton, Radin, and Sadun show in [16] that the stretching factor of any other similarity which generates $\mathcal{T}$ is equal to a rational power of $\lambda_{1}$. More precisely, they prove that the stretching factors of conjugate tiling systems which are the orbit closure under Euclidean motions of some self-similar tilings are multiplicatively dependent. In this paper we look at tiling systems which are the orbit closure under translations of some self-similar tilings, in order to give a necessary condition to have nonperiodic common factors. The result we present in this paper is the following:

\footnotetext{
M.I. Cortez

Departamento de Matemática, y CC. de la Universidad de Santiago de Chile, Av. Libertador Bernardo O’Higgins 3363, Santiago, Chile

e-mail: mcortez@usach.cl

F. Durand $(\bowtie)$

Laboratoire Amiénois de Mathématiques Fondamentales et Appliquées, CNRS-UMR 6140, Université de Picardie Jules Verne, 33 rue Saint Leu, 80039 Amiens Cedex, France

e-mail: fabien.durand@u-picardie.fr
} 
Theorem 1 Let $S_{1}\left(\mathcal{T}_{1}\right)=\mathcal{T}_{1}$ and $S_{2}\left(\mathcal{T}_{2}\right)=\mathcal{T}_{2}$ be two self-similar tilings satisfying the Finite Pattern Condition, where $S_{1}$ and $S_{2}$ are primitive substitutions. Let $\lambda_{1}$ and $\lambda_{2}$ be the Perron eigenvalues of the substitution matrices associated to $S_{1}$ and $S_{2}$, respectively. If there exist a nonperiodic tiling $\mathcal{T}$ and factor maps $\pi_{i}: \Omega_{\mathcal{T}_{i}} \rightarrow \Omega_{\mathcal{T}}$ for $i \in\{1,2\}$, then $\lambda_{1}$ and $\lambda_{2}$ are multiplicatively dependent.

The problem we are interested in has been considered a long time ago by Cobham in [5] and [6] for fixed points of substitutions of constant length. He showed that if $p, q>1$ are two multiplicatively independent integers, then a sequence $x$ on a finite alphabet is both $p$-substitutive and $q$-substitutive if and only if $x$ is ultimately periodic, where $p$-substitutive means that $x$ is the image by a letter-to-letter morphism of a fixed point of a substitution of constant length $p$. This theorem was the starting point of a lot of work in many different directions such as: numeration systems for $\mathbb{N}$, substitutive sequences and subshifts, automata theory and logic (for more details, see [1-4, 7-14, 17]). Later, Semenov [21] proved a "multidimensional" Cobham-type theorem, that is to say, a Cobham theorem for recognizable subsets of $\mathbb{N}^{d}$. This result can be stated in terms of self-similar tilings, and in the case these tilings are repetitive, our result is a generalization of Semenov Theorem.

This paper is organized as follows: in Sect. 2 we give some basic definitions relevant for the study of tiling systems and substitution tiling systems. In Sect. 3 we study the frequencies of the patches in self-similar tilings and in their factors. First we prove that the frequencies of the patches in a self-similar tiling $\mathcal{T}$ are included in a finite union of geometric progressions of rate $\lambda$, where $\lambda$ is the stretching factor of $\mathcal{T}$ (in [15] the authors remarked this fact for minimal substitution subshifts). Next, we prove that the frequencies of the patches in a tiling $\mathcal{T}$, which is a factor of two selfsimilar tiling systems with stretching factors $\lambda_{1}$ and $\lambda_{2}$, respectively, are included in the intersection of two finite unions of geometric progressions, one of rate $\lambda_{1}$ and the other of rate $\lambda_{2}$. The proof of this result would be easier if the factor maps were given by a kind of "sliding block code" (as it can be the case for subshifts), because in this case the preimage of a patch would be a finite collection of patches. Nevertheless, this is no longer the case for the tiling systems we consider here (examples of factor maps, and even conjugacies, that are not given by a "sliding block codes" are given in [18] and [19]), but we overcome this problem selecting carefully some patches in the preimages we considered. Finally, in Sect. 4 we deduce the main theorem.

\section{Definitions and Background}

In this section we give the classical definitions concerning tilings. For more details, we refer to [22]. A tiling of $\mathbb{R}^{d}$ is a countable collection $\mathcal{T}=\left\{t_{i}: i \geq 0\right\}$ of closed subsets of $\mathbb{R}^{d}$ (which are known as tiles) whose union is the whole space and their interiors are pairwise disjoint. We assume that the tiles are homeomorphic to closed balls and that they belong, up to translations, to a finite collection of closed subsets of $\mathbb{R}^{d}$ whose elements are called prototiles. We say that two tiles are equivalent if they are equal up to translations. It is often useful to consider every prototile as a closed set endowed with a label. In this case, two tiles are equivalent if, in addition, their labels coincide. 
The translation of the tiling $\mathcal{T}$ by a vector $v \in \mathbb{R}^{d}$ is the tiling $\mathcal{T}+v$ obtained after translating every tile of $\mathcal{T}$ by $-v$. The tiling $\mathcal{T}$ is said to be aperiodic (or nonperiodic) if $\mathcal{T}+v=\mathcal{T}$ implies $v=0$.

The support of a tile $t_{i}$, denoted by $\operatorname{supp}\left(t_{i}\right)$, is the closed set that defines $t_{i}$. For every subset $A$ of $\mathbb{R}^{d}$, we define, as usual, $\mathcal{T} \cap A$ to be the set $\left\{t_{i} \cap A: i \geq 0\right\}$. A patch $P$ is a finite collection of tiles. The support of a patch $P$, denoted by $\operatorname{supp}(P)$, is the union of the supports of the tiles in $P$. The diameter of a patch $P$ is the diameter of its support, we call it $\operatorname{diam}(P)$. We define $P+v$ as we defined $\mathcal{T}+v$.

The tiling $\mathcal{T}$ satisfies the finite pattern condition FPC (or equivalently, we say that it is locally finite) if for any $r>0$, there are up to translation, only finitely many patches with diameter smaller than $r$. This condition is automatically satisfied in the case of a tiling whose tiles are polyhedra that meet face-to-face. A tiling $\mathcal{T}$ is repetitive if for any patch $P$ in $\mathcal{T}$, there exists $r>0$ such that for every open ball $B_{r}(v)$, the collection $\mathcal{T} \cap B_{r}(v)$ contains a patch $P^{\prime}$ equivalent to $P$ (when it is clear from the context, we will say that $P$ "appears" in $B_{r}(v)$ ). Nonperiodic repetitive tilings that satisfy FPC are called perfect tilings.

\subsection{Tiling Systems}

Let $\mathcal{A}$ be a finite collection of prototiles. We denote by $T(\mathcal{A})$ (full tiling space) the space of all tilings of $\mathbb{R}^{d}$ whose tiles are equivalent to some element in $\mathcal{A}$. We always suppose that $T(\mathcal{A})$ is nonempty. The group $\mathbb{R}^{d}$ acts on $T(\mathcal{A})$ by translations:

$$
(v, \mathcal{T}) \rightarrow \mathcal{T}+v \quad \text { for } v \in \mathbb{R}^{d} \text { and } \mathcal{T} \in T(\mathcal{A}) .
$$

Furthermore, this action is continuous with the topology induced by the following distance: take $\mathcal{T}, \mathcal{T}^{\prime}$ in $T(\mathcal{A})$ and define $A$ the set of $\varepsilon \in(0,1)$ such that there exist $v$ and $v^{\prime}$ in $B_{\varepsilon}(0)$ with

$$
(\mathcal{T}+v) \cap B_{1 / \varepsilon}(0)=\left(\mathcal{T}^{\prime}+v^{\prime}\right) \cap B_{1 / \varepsilon}(0) ;
$$

we set

$$
d\left(\mathcal{T}, \mathcal{T}^{\prime}\right)= \begin{cases}\inf A & \text { if } A \neq \emptyset \\ 1 & \text { if } A=\emptyset\end{cases}
$$

Roughly speaking, two tilings are close if they have the same pattern in a large neighborhood of the origin, up to a small translation. A tiling system is a pair $\left(\Omega, \mathbb{R}^{d}\right)$ such that $\Omega$ is a translation invariant closed subset of some full tiling space. The orbit closure of a tiling $\mathcal{T}$ in $T(\mathcal{A})$ is the set $\Omega_{\mathcal{T}}=\overline{\left\{\mathcal{T}+v: v \in \mathbb{R}^{d}\right\}}$. When $\mathcal{T}$ satisfies the FPC, $\Omega_{\mathcal{T}}$ is compact (see [20]). If $\mathcal{T}$ is repetitive, then all the orbits are dense in $\Omega_{\mathcal{T}}$. In this case the tiling system $\left(\Omega_{\mathcal{T}}, \mathbb{R}^{d}\right)$ is said to be minimal.

A factor map between two tiling systems $\left(\Omega_{1}, \mathbb{R}^{d}\right)$ and $\left(\Omega_{2}, \mathbb{R}^{d}\right)$ is a continuous map $\pi: \Omega_{1} \rightarrow \Omega_{2}$ such that $\pi(\mathcal{T}+v)=\pi(T)+v$ for all $\mathcal{T} \in \Omega_{1}$ and $v \in \mathbb{R}^{d}$.

In symbolic dynamics it is well known that topological factor maps between subshifts are always given by sliding-block-codes. There are examples which show that this result cannot be extended to tiling systems $[18,19]$. The following lemma shows that factor maps between tiling systems are not far to be sliding-block-codes. A similar result can be found in [16]. 
Lemma 2 Let $\mathcal{T}_{1}$ and $\mathcal{T}_{2}$ be two tilings. Suppose that $\mathcal{T}_{1}$ verifies the FPC and $\pi$ : $\Omega_{\mathcal{T}_{1}} \rightarrow \Omega_{\mathcal{T}_{2}}$ is a factor map. Then, there exists a constant $s_{0}>0$ such that to every $\varepsilon>0$ it is possible to associate $R_{\varepsilon}>0$ satisfying the following: Let $R \geq R_{\varepsilon}$. If $\mathcal{T}$ and $\mathcal{T}^{\prime}$ in $\Omega_{\mathcal{T}_{1}}$ verify

$$
\mathcal{T} \cap B_{R+s_{0}}(0)=\mathcal{T}^{\prime} \cap B_{R+s_{0}}(0)
$$

then

$$
(\pi(\mathcal{T})+v) \cap B_{R}(0)=\pi\left(\mathcal{T}^{\prime}\right) \cap B_{R}(0)
$$

for some $v \in B_{\varepsilon}(0)$.

Proof The tiling $\mathcal{T}_{2}$ also satisfies the FPC because $\Omega_{\mathcal{T}_{2}}$ is compact. Since the tilings in $\Omega_{\mathcal{T}_{2}}$ have a finite number of tiles, up to translations, there exists $\delta_{0}^{\prime}>0$ such that if $y_{1} \neq y_{2} \in \mathbb{R}^{d}$ satisfy $\left(\mathcal{T}+y_{1}\right) \cap B_{R}(0)=\left(\mathcal{T}+y_{2}\right) \cap B_{R}(0)$ for some $\mathcal{T} \in \Omega_{\mathcal{T}_{2}}$ and some $R>\max \{\operatorname{diam}(p): p$ prototile in $\mathcal{T}\}$, then $\left\|y_{1}-y_{2}\right\| \geq \delta_{0}^{\prime}$ (for the details, see [22]).

Let $0<\delta_{0}<\frac{\delta_{0}^{\prime}}{2}$. Since $\pi$ is uniformly continuous, there exists $s_{0}>1$ such that if $\mathcal{T}$ and $\mathcal{T}^{\prime}$ in $\Omega_{\mathcal{T}_{1}}$ verify $\mathcal{T} \cap B_{s_{0}}(0)=\mathcal{T}^{\prime} \cap B_{s_{0}}(0)$, then

$$
(\pi(\mathcal{T})+v) \cap B_{\frac{1}{\delta_{0}}}(0)=\pi\left(\mathcal{T}^{\prime}\right) \cap B_{\frac{1}{\delta_{0}}}(0)
$$

for some $v \in B_{\delta_{0}}(0)$.

Let $0<\varepsilon<\delta_{0}$. By the uniform continuity of $\pi$ there exists $0<\delta<\frac{1}{s_{0}}$ such that if $\mathcal{T}$ and $\mathcal{T}^{\prime}$ in $\Omega_{\mathcal{T}_{1}}$ verify $\mathcal{T} \cap B_{\frac{1}{\delta}}(0)=\mathcal{T}^{\prime} \cap B_{\frac{1}{\delta}}(0)$, then

$$
(\pi(\mathcal{T})+v) \cap B_{\frac{1}{\varepsilon}}(0)=\pi\left(\mathcal{T}^{\prime}\right) \cap B_{\frac{1}{\varepsilon}}(0)
$$

for some $v \in B_{\varepsilon}(0)$.

Now fix $R \geq R_{\varepsilon}=\frac{1}{\delta}-s_{0}$ and $\mathcal{T}$ and $\mathcal{T}^{\prime}$ two tilings in $\Omega_{\mathcal{T}_{1}}$ verifying

$$
\mathcal{T} \cap B_{R+s_{0}}(0)=\mathcal{T}^{\prime} \cap B_{R+s_{0}}(0) .
$$

Then, on one hand, the tilings $\mathcal{T}$ and $\mathcal{T}^{\prime}$ satisfy (2.1), and, on the other hand, we obtain that $(\mathcal{T}+a) \cap B_{s_{0}}(0)=\left(\mathcal{T}^{\prime}+a\right) \cap B_{s_{0}}(0)$ for every $a$ in $B_{R}(0)$. By the choice of $s_{0}$ this implies that

$$
\left(\pi(\mathcal{T})+a+t_{a}\right) \cap B_{\frac{1}{\delta_{0}}}(0)=\left(\pi\left(\mathcal{T}^{\prime}\right)+a\right) \cap B_{\frac{1}{\delta_{0}}}(0)
$$

for some $t_{a} \in B_{\delta_{0}}(0)$.

Since $\delta_{0}>\varepsilon$, from (2.1) we get

$$
(\pi(\mathcal{T})+v) \cap B_{\frac{1}{\delta_{0}}}(0)=\pi\left(\mathcal{T}^{\prime}\right) \cap B_{\frac{1}{\delta_{0}}}(0) .
$$

We will show that $t_{a}=v$ for every $a$ in $B_{R}(0)$. This property, together with (2.3) and (2.4), implies that

$$
(\pi(\mathcal{T})+v) \cap B_{R}(0)=\pi\left(\mathcal{T}^{\prime}\right) \cap B_{R}(0) .
$$


For $a=0$, from (2.3) and (2.4) we have that $t_{0}=v$ or $\left\|v-t_{0}\right\| \geq \delta_{0}^{\prime}$. Since $\left\|t_{0}-v\right\| \leq$ $\delta_{0}+\varepsilon<2 \delta_{0}<\delta_{0}^{\prime}$, we conclude that $t_{0}=v$.

For $a \in B_{R}(0)$, consider $s>0$ such that for every $a^{\prime} \in B_{S}(a)$, the patch

$$
P=\left(\left(\pi\left(\mathcal{T}^{\prime}\right)+a\right) \cap B_{\frac{1}{\delta_{0}}}(0)\right) \cap\left(\left(\pi\left(\mathcal{T}^{\prime}\right)+a+\left(a^{\prime}-a\right)\right) \cap B_{\frac{1}{\delta_{0}}}(0)\right)
$$

contains a tile.

From (2.3) we get $\pi(\mathcal{T})+a+t_{a}+\left(a-a^{\prime}\right) \cap \operatorname{supp}(P)=P$. Replacing $a$ by $a^{\prime}$ in (2.3), we obtain $\pi(\mathcal{T})+a+t_{a}^{\prime}+\left(a^{\prime}-a\right) \cap \operatorname{supp}(P)=P$. This implies that the norm of $t_{a}-t_{a}^{\prime}$ is equal to 0 or greater than $\delta_{0}^{\prime}$. Since $\left\|t_{a}-t_{a}^{\prime}\right\| \leq 2 \delta_{0}<\delta_{0}^{\prime}$, we get $t_{a}=t_{a}^{\prime}$. Thus we conclude that the function that associates $t_{a}$ to $a$ is constant, which implies that $t_{a}=t_{0}=v$ for every $a$ in $B_{R}(0)$.

\subsection{Linearly Recurrent Tilings}

A tiling $\mathcal{T}$ is linearly recurrent (or strongly repetitive, or linearly repetitive) if there exists a constant $L>0$ such that for every patch $P$ in $\mathcal{T}$, any ball of radius $L \operatorname{diam}(P)$ contains a translate of $P$. Every tiling in the orbit closure of a linearly recurrent tiling is linearly recurrent with the same constant. When $\mathcal{T}$ is linearly recurrent, we call $\left(\Omega_{\mathcal{T}}, \mathbb{R}^{d}\right)$ a linearly recurrent tiling system.

Lemma 3 Let $\mathcal{T}_{1}$ and $\mathcal{T}_{2}$ be two tilings verifying the FPC. If $\pi: \Omega_{\mathcal{T}_{1}} \rightarrow \Omega_{\mathcal{T}_{2}}$ is a factor map and $\mathcal{T}_{1}$ is linearly repetitive, then $\left(\Omega_{\mathcal{T}_{2}}, \mathbb{R}^{d}\right)$ is linearly recurrent.

Proof Let $\mathcal{T} \in \Omega_{\mathcal{T}_{1}}$. Consider $\varepsilon>0$ and $R>0$ the positive number of Lemma 2 associated to $\varepsilon$. Since $\mathcal{T}$ is linearly repetitive with some constant $L$, for any $y \in$ $\mathbb{R}^{d}$, there exists $v \in B_{L\left(R+s_{0}\right)}(y)$ such that $B_{R+s_{0}}(v) \subseteq B_{L\left(R+s_{0}\right)}(y)$ and $(\mathcal{T}+v) \cap$ $B_{R+s_{0}}(0)=\mathcal{T} \cap B_{R+s_{0}}(0)$. By Lemma 2 there exists $t \in B_{\varepsilon}(0)$ such that $(\pi(\mathcal{T})+$ $v+t) \cap B_{R}(0)=\pi(\mathcal{T}) \cap B_{R}(0)$. This implies that any ball of radius $L\left(R+s_{0}\right)+2 \varepsilon$ in $\pi(\mathcal{T})$ contains a copy of $\pi(\mathcal{T}) \cap B_{R}(0)$. Since $L s_{0}+2 \varepsilon$ is smaller than some constant, it follows that $\pi(\mathcal{T})$ is linearly recurrent.

\subsection{Substitution Tiling Systems}

Let $M$ be a linear map on $\mathbb{R}^{d}$. It is called expansive if there exists $\lambda>1$ such that

$$
\|M v\| \geq \lambda\|v\| \quad \text { for all } v \in \mathbb{R}^{d} .
$$

The map $M$ is a similarity if $\|M v\|=\lambda\|v\|$ for all $v \in \mathbb{R}^{d}$.

Let $\alpha$ be an eigenvalue of the expansive (resp. similar) linear map $M$, and let $v \neq 0$ be an eigenvector associated to $\alpha$. We have $\|M v\|=|\alpha|\|v\|$, which implies that $|\alpha| \geq \lambda$ (resp. $|\alpha|=\lambda$ ) and then $|\operatorname{det}(M)| \geq \lambda^{d}\left(\operatorname{resp} .|\operatorname{det}(M)|=\lambda^{d}\right)$. Thus, if $\Theta$ is a Borel set in $\mathbb{R}^{d}$, we obtain

$$
\begin{array}{ll}
\operatorname{vol}(M \Theta)=|\operatorname{det}(M)| \operatorname{vol}(\Theta) \geq \lambda^{d} \operatorname{vol}(\Theta) & \text { if } M \text { is expansive, } \\
\operatorname{vol}(M \Theta)=|\operatorname{det}(M)| \operatorname{vol}(\Theta)=\lambda^{d} \operatorname{vol}(\Theta) & \text { if } M \text { is a similarity. }
\end{array}
$$


Let $\mathcal{A}$ be a finite collection of prototiles, and let $M$ be an expansive linear map on $\mathbb{R}^{d}$. A substitution is a function $S$ on the set of prototiles $\mathcal{A}$ that associates to each $p$ in $P$ a patch $S(p)$ such that

- the support of $S(p)$ is $M \operatorname{supp}(p)$,

- for every $q \in \mathcal{A}$, there exist $n_{p, q} \geq 0$ and, for each $1 \leq k \leq n_{p, q}, v_{p, q, k} \in \mathbb{R}^{d}$ such that

$$
S(p)=\left\{q+v_{p, q, k}: 1 \leq k \leq n_{p, q}, q \in \mathcal{A}\right\} .
$$

The substitution matrix of $S$ is the matrix $A \in \mathcal{M}_{\mathcal{A} \times \mathcal{A}}\left(\mathbb{Z}^{+}\right)$which contains, in the coordinate $(p, q)$, the number of different tiles in $S(p)$ which are equivalent to $q$. That is, $A_{p, q}=n_{p, q}$ for all $p, q \in \mathcal{A}$.

The substitution $S$ can be defined on $T(\mathcal{A})$ in the following way: if $t$ is a tile in $\mathcal{T} \in T(\mathcal{A})$ such that $t$ is equivalent to the prototile $p \in \mathcal{A}$, we define

$$
S(t)=S(p)+M v,
$$

where $v \in \mathbb{R}^{d}$ is such that $\operatorname{supp}(t)=\operatorname{supp}(p)+v$. Then, we define

$$
S(\mathcal{T})=\bigcup_{t \in \mathcal{T}} S(t) \in T(\mathcal{A})
$$

The substitution is primitive if $A$ is primitive, that is, there exists $k>0$ such that $A^{k}>0$. In this case, the Perron eigenvalue of $A$ is $|\operatorname{det}(M)|[22]$.

In this paper, we always suppose that $S$ is primitive.

The substitution tiling system associated to $S$ is the tiling system $\left(X_{S}, \mathbb{R}^{d}\right)$, where $X_{S}$ is the space of all tilings $\mathcal{T}$ in $T(\mathcal{A})$ such that for every patch $P$ of $\mathcal{T}$, there exist a prototile $p \in \mathcal{A}$ and $k>0$ satisfying $P \subseteq S^{k}(p)$. The action of $\mathbb{R}^{d}$ on $X_{S}$ is the translation. Because $S$ is primitive, there always exist a tiling $\mathcal{T}_{0} \in T(\mathcal{A})$ and $k_{0}>0$ such that $S^{k_{0}}\left(\mathcal{T}_{0}\right)=\mathcal{T}_{0}$. It is classical (in the primitive case) that $\Omega_{\mathcal{T}_{0}}=X_{S}=X_{S^{k}}$ for every $k>0$. So, without loss of generality we can suppose that $S\left(\mathcal{T}_{0}\right)=\mathcal{T}_{0}$. In addition, we will always suppose that the fixed point of $S$ satisfies the FPC. In this case, $X_{S}$ is a compact metric space, and $\left(X_{S}, \mathbb{R}^{d}\right)$ is minimal.

A tiling $\mathcal{T}$ in $T(\mathcal{A})$ which satisfies the FPC is self-affine if it is the fixed point of a substitution. The tiling $\mathcal{T}$ is said to be self-similar if it is the fixed point of a substitution $S$ which is defined by a similarity $M$ with constant $\lambda$ (for more details, see [22]). We say that $\lambda$ is the stretching factor of $S$ or $\mathcal{T}$.

Let $\mathcal{T}_{0}$ be a self-similar tiling which is the fixed point of a primitive substitution $S$ satisfying the FPC. The following two results are included in [23].

Lemma $4 \mathcal{T}_{0}$ is linearly recurrent.

Lemma 5 There exists $N>0$ such that if $P$ is a patch in $\mathcal{T}_{0}$ whose support contains a ball of radius $R$, then whenever $P+v$ is a patch of $\mathcal{T}_{0}$ with $v>0,\|v\|>\frac{R}{N}$.

These two lemmata mean that the minimal distance between two equivalent patches in a self-similar tiling is neither too large nor too small compared to their sizes. 


\section{Frequencies}

Consider a tiling $\mathcal{T}$ of $\mathbb{R}^{d}$. For a set $F \subseteq \mathbb{R}^{d}$, we write

$$
\mathcal{T} \llbracket F \rrbracket=\{t \in \mathcal{T}: t \cap F \neq \emptyset\} .
$$

A $\mathcal{T}$-corona is a patch $\mathcal{T} \llbracket \operatorname{supp}(t) \rrbracket$, where $t$ is a tile in $\mathcal{T}$. Remark that for some $\epsilon \in \mathbb{R}^{d}$, we could have $\mathcal{T} \llbracket F+\epsilon \rrbracket=\mathcal{T} \llbracket F \rrbracket$. To avoid this situation we define, for $v \in \mathbb{R}^{d}, \mathcal{T}[F, v]=\mathcal{T} \llbracket F \rrbracket-v$. When $F$ is a ball $B_{R}(v)$, we write $\mathcal{T}\left[B_{R}(v)\right]$ instead of $\mathcal{T}\left[B_{R}(v), v\right]$.

In the sequel we suppose that $\mathcal{T}_{0}$ is a self-similar tiling which is the fixed point of a primitive substitution $S$, with stretching factor $\lambda$, satisfying the FPC.

\subsection{Van Hove Sequences}

In order to define the notion of frequency of a patch, we need the concept of Van Hove sequences.

Let $P$ be a patch in $\mathcal{T}_{0}$, and let $\Theta \subset \mathbb{R}^{d}$. Denote by $L_{P}(\Theta)$ the number of patches included in $\mathcal{T}_{0} \cap \Theta$ which are equivalent to $P$ [22].

A sequence $\left(\Theta_{n}\right)_{n \geq 0}$ of subsets of $\mathbb{R}^{d}$ is a Van Hove sequence if for any $r>0$,

$$
\lim _{n \rightarrow \infty} \frac{\operatorname{vol}\left(\left(\partial \Theta_{n}\right)^{+r}\right)}{\operatorname{vol}\left(\Theta_{n}\right)}=0
$$

where

$$
\Theta^{+r}=\left\{x \in \mathbb{R}^{d}: \operatorname{dist}(x, \Theta) \leq r\right\},
$$

and $\partial \Theta$ is the border of $\Theta$.

In [22], it was shown that for any patch $P$ in $\mathcal{T}_{0}$, there is a number $\operatorname{freq}(P)>0$ such that for any Van Hove sequence $\left(\Theta_{n}\right)_{n \geq 0}$,

$$
\lim _{n \rightarrow \infty} \frac{L_{P}\left(\Theta_{n}\right)}{\operatorname{vol}\left(\Theta_{n}\right)}=\operatorname{freq}(P) .
$$

Suppose that $P$ and $Q$ are two patches in $\mathcal{T}_{0}$. In order to simplify the notation, we will write $L_{P}(Q), \operatorname{vol}(P)$ and $(\partial P)^{+r}$ instead of $L_{P}(\operatorname{supp}(Q)), \operatorname{vol}(\operatorname{supp}(P))$ and $(\partial \operatorname{supp}(P))^{+r}$ respectively.

It is easy to show that $\left(M^{n} \Theta\right)_{n \geq 0}$ is a Van Hove sequence when $M: \mathbb{R}^{d} \rightarrow \mathbb{R}^{d}$ is an expansive linear map and $\Theta$ is a compact subset of $\mathbb{R}^{d}$ with nonempty interior and such that $\operatorname{vol}(\partial \Theta)=0$. Consequently, to compute freq $(P)$ we will use the limit

$$
\operatorname{freq}(P)=\lim _{k \rightarrow \infty} \frac{L_{P}\left(S^{k}(p)\right)}{\operatorname{vol}\left(S^{k}(p)\right)}
$$

for any prototile $p$ in $\mathcal{A}$. 


\subsection{Patch Frequencies of a Self-Similar Tiling}

The next proposition extends a result of C. Holton and L. Zamboni [15] obtained for minimal substitution subshifts. But before we will need the following technical lemma:

Lemma 6 Suppose that $\mathcal{T}$ satisfies the FPC. Then there exists a constant $\eta>0$ such that for every $y \in \mathbb{R}^{d}$, the ball $B_{\eta}(y)$ is contained in the support of a corona in $\mathcal{T}$.

Proof Let $t$ be a tile in $\mathcal{T}$. The number

$$
\eta_{t}=\operatorname{dist}(\partial t, \partial \mathcal{T} \llbracket[\operatorname{supp}(t)])
$$

is positive for every tile $t$. The FPC implies that there is a finite number of coronas up translations. Hence we get

$$
\eta=\min \left\{\eta_{t}: t \in \mathcal{T}\right\}>0 .
$$

Notice that the set

$$
\left\{x \in \mathbb{R}^{d}: \operatorname{dist}(x, t) \leq \eta\right\}
$$

is contained in the support of $\mathcal{T} \llbracket \operatorname{supp}(t) \rrbracket$ for every tile $t$ in $\mathcal{T}$. Thus if $y$ is a point in $\mathbb{R}^{d}$ belonging to the tile $t \in \mathcal{T}$, then the ball $B_{\eta}(y)$ is contained in the support of $\mathcal{T} \llbracket \operatorname{supp}(t) \rrbracket$.

Proposition 7 There exists a finite set $F \subset \mathbb{R}$ such that for every patch $P$ in $\mathcal{T}_{0}$ satisfying $P=\mathcal{T}_{0}\left[B_{R}(y)\right]$, for some $R>0$ and $y \in \mathbb{R}^{d}$,

$$
\operatorname{freq}(P)=\frac{f}{\lambda^{d k}},
$$

where $f \in F$ and $k>0$ is such that

$$
\lambda^{k-1} \eta \leq \operatorname{diam}(P)<\lambda^{k} \eta
$$

with $\eta$ the constant of Lemma 6.

Proof Let $\mathcal{A}$ be the prototile set associated to $\mathcal{T}_{0}$. We define

$$
\bar{l}=\max \{\operatorname{diam}(p): p \in \mathcal{A}\} .
$$

Let $P$ be a patch in $\mathcal{T}_{0}$ such that

$$
\left.P=\mathcal{T}_{0} \llbracket B_{R}(y)\right] \quad \text { for some } R>0 \text { and } y \in \mathbb{R}^{d} .
$$

This implies that

$$
\operatorname{diam}(P) \leq 2(R+\bar{l})
$$


Let $k \geq 0$ be such that

$$
\lambda^{k-1} \eta \leq \operatorname{diam}(P)<\lambda^{k} \eta
$$

By Lemma 6, there exists a corona $B$ the support of which contains the ball $B_{\eta}\left(M^{-k} y\right)$. Because the support of $S^{k}(B)$ contains the ball $B_{\lambda^{k} \eta}(y)$, by (3.2) we deduce that $S^{k}(B)$ contains the patch $P$. From Lemma 5 we have

$$
L_{P}\left(S^{k}(B)\right) \leq \frac{\operatorname{vol}\left(S^{k}(B)\right)}{\operatorname{vol}\left(B_{\frac{R}{N}}(0)\right)}=\frac{\lambda^{k d}}{\frac{R^{d}}{N^{d}}} \frac{\operatorname{vol}(B)}{\operatorname{vol}\left(B_{1}(0)\right)}
$$

From (3.1) and (3.2) we obtain

$$
\frac{1}{2(R+\bar{l})} \leq \frac{1}{\operatorname{diam}(P)} \leq \frac{1}{\lambda^{k-1} \eta}
$$

which implies that there exists $C$, independent of $k$, such that

$$
\frac{\lambda^{k d}}{R^{d}} \leq\left(\frac{2 \lambda}{\eta-\frac{2 \bar{l}}{\lambda^{k-1}}}\right)^{d} \leq C .
$$

From (3.3) and (3.4) we conclude that there exists a constant $K$, independent on $P$, $k$ and $B$, such that

$$
L_{P}\left(S^{k}(B)\right) \leq K
$$

Let $P^{\prime}$ be any patch in $\mathcal{T}_{0}$, and let $D$ be the set of all $\mathcal{T}_{0}$-coronas, up to translation. We have

$$
L_{P}\left(S^{k}\left(P^{\prime}\right)\right)=\sum_{B \in D} L_{B}\left(P^{\prime}\right) N\left(P^{\prime}, P, B\right),
$$

where $N\left(P^{\prime}, P, B\right)$ is some integer in $\left\{0, \ldots, L_{P}\left(S^{k}(B)\right)\right\} \subseteq\{0, \ldots, K\}$. Thus, for $p \in \mathcal{A}$ and $n>k$,

$$
\begin{aligned}
\frac{L_{P}\left(S^{n}(p)\right)}{\operatorname{vol}\left(S^{n}(p)\right)} & =\frac{L_{P}\left(S^{k}\left(S^{n-k}(p)\right)\right)}{\operatorname{vol}\left(S^{n}(p)\right)} \\
& =\sum_{B \in D} \frac{L_{B}\left(S^{n-k}(p)\right) N\left(S^{k-n}(p), P, B\right)}{\operatorname{vol}\left(S^{n}(p)\right)} \\
& =\sum_{B \in D} \frac{L_{B}\left(S^{n-k}(p)\right)}{\operatorname{vol}\left(S^{n-k}(p)\right)} \frac{\operatorname{vol}\left(S^{n-k}(p)\right)}{\operatorname{vol}\left(S^{n}(p)\right)} N\left(S^{k-n}(p), P, B\right) \\
& =\frac{1}{\lambda^{k d}} \sum_{B \in D} \frac{L_{B}\left(S^{n-k}(p)\right)}{\operatorname{vol}\left(S^{n-k}(p)\right)} N\left(S^{k-n}(p), P, B\right) .
\end{aligned}
$$


Because $N\left(S^{k-n}(p), P, B\right)$ is in $\{1, \ldots, K\}$ for every $n>k$, we can take a convergent subsequence to obtain

$$
\begin{aligned}
\operatorname{freq}(P) & =\frac{1}{\lambda^{k d}} \lim _{n \rightarrow \infty} \sum_{B \in D} \frac{L_{B}\left(S^{n-k}(p)\right)}{\operatorname{vol}\left(S^{n-k}(p)\right)} N\left(S^{k-n}(p), P, B\right) \\
& =\frac{1}{\lambda^{k d}} \sum_{B \in D} \operatorname{freq}(B) N(P, B),
\end{aligned}
$$

where $N(P, B)$ is some integer in $\{0, \ldots, K\}$ for every $B \in D$. Because $D$ is finite, to conclude it suffices to take

$$
F=\left\{\sum_{B \in D} \operatorname{freq}(B) N_{B}: N_{B} \in\{0, \ldots, K\}\right\} .
$$

Remark 8 From [22] we know that $\left(\Omega_{\mathcal{T}_{0}}, \mathbb{R}^{d}\right)$ is uniquely ergodic. Hence, the frequency of a patch $P$ does not depend on the tiling. That is, $\operatorname{freq}(P)$ is the same for every $\mathcal{T}$ in $\Omega_{\mathcal{T}_{0}}$.

\subsection{Patch Frequency in the Factor}

The next result extends Proposition 7 to tiling factors of self-similar tiling systems. The main problem we have to overcome is that the factor map is not necessarily given by a sliding block code. Hence the first part of the next proof consists in selecting carefully the preimages of a given patch $P$ by means of a finite induction procedure. Then, we show that the frequency of the patch $P$ is the sum of the frequencies of the selected patches.

Proposition 9 Let $\mathcal{T}$ be a nonperiodic tiling. If there exists a factor map $\pi: \Omega_{\mathcal{T}_{0}} \rightarrow$ $\Omega_{\mathcal{T}}$, then there exists a finite set $F \subseteq \mathbb{R}$ such that for every patch $P$ in $\mathcal{T}$ satisfying $P=\mathcal{T}\left[B_{R}(y)\right]$, for some $R>0$ and $y \in \mathbb{R}^{d}$,

$$
\operatorname{freq}(P)=\frac{f}{\lambda^{d k}},
$$

where $f \in F$ and $k>0$ is such that

$$
\eta \lambda^{k-3} \leq \operatorname{diam}(P)<\eta \lambda^{k-1}
$$

if $R$ is large enough.

Proof Let $\mathcal{T}_{2} \in \Omega_{\mathcal{T}}$, and let $\mathcal{T}_{1} \in \Omega_{\mathcal{T}_{0}}$ be such that $\pi\left(\mathcal{T}_{1}\right)=\mathcal{T}_{2}$. Let $s_{0}>0$ be the constant of Lemma 2.

The linear recurrence of $\mathcal{T}_{1}$ implies that the tiling $\mathcal{T}_{2}$ is also linearly recurrent. Let $L$ be the constant of linear recurrence of $\mathcal{T}_{1}$, and let $M$ and $N$ be the constants of Lemma 5 associated to $\mathcal{T}_{1}$ and $\mathcal{T}_{2}$, respectively. We set

$$
K=\max \left\{(8 L N)^{d},(8 L M)^{d}\right\}
$$


and

$$
\eta_{i}=\max \left\{\operatorname{diam}(t): t \text { is a tile in } \mathcal{T}_{i}\right\} \quad \text { for } i \in\{1,2\} .
$$

Let $\varepsilon>0$. Let $R_{\varepsilon}>0$ be the positive number associated to $\varepsilon$ as in Lemma 2. Notice that $R_{\varepsilon}$ can be chosen large enough in order that

$$
R_{\varepsilon} \geq \max \left\{\begin{array}{l}
s_{0}+\eta_{1}+\eta_{2}+\varepsilon, \\
4 N(2 K+1) \varepsilon, \\
2 M \varepsilon-s_{0}, \\
2\left(\eta_{1}+\varepsilon\right)-\left(s_{0}+\eta_{2}\right), \\
\eta \lambda^{\left\lceil\log _{\lambda} \frac{2 \eta_{1}}{\eta(\lambda-1)}\right\rceil}, \\
\eta \lambda^{\left\lceil\log _{\lambda} \frac{2\left(s_{0}+\eta_{1}+\eta_{2}+2 \varepsilon\right)}{\eta(\lambda-1)}\right\rceil+2} \\
\eta / 2 .
\end{array}\right.
$$

Let $R \geq R_{\varepsilon}$, and let $P=\mathcal{T}_{2}\left[B_{R}(y)\right], y \in \mathbb{R}^{d}$.

Suppose that $v_{1}, \ldots, v_{l}$ are all the points in $B_{2 L\left(R+s_{0}+\varepsilon+\eta_{1}+\eta_{2}\right)}(0)$ such that

$$
\mathcal{T}_{2}\left[B_{R}\left(v_{i}\right)\right]=P \text {. }
$$

If $v_{i} \neq v_{j}$, we have $\left\|v_{i}-v_{j}\right\|>\frac{R}{N}$. This implies that in a ball of radius $\frac{R}{2 N}$, there is at most one point $v$ such that $\mathcal{T}_{2}\left[B_{R}(v)\right]=P$. Using (3.5), it follows that in $B_{2 L\left(R+s_{0}+\varepsilon+\eta_{1}+\eta_{2}\right)}(0)$ there are at most

$$
\frac{\operatorname{vol}\left(B_{2 L\left(R+s_{0}+\varepsilon+\eta_{1}+\eta_{2}\right)}(0)\right)}{\operatorname{vol}\left(B_{\frac{R}{2 N}}(0)\right)} \leq(8 L N)^{d} \leq K
$$

points $v$ such that $\mathcal{T}_{2}\left[B_{R}(v)\right]=P$. This implies that for any patch $P$, we have $l \leq K$.

For every $1 \leq i \leq l$, we set

$$
P_{i}=\mathcal{T}_{1}\left[B_{R+s_{0}+\eta_{2}}\left(v_{i}\right)\right]
$$

Now, for every $1 \leq i \leq l$, we will define, by induction on $i, k_{i}$ different patches as follows (see Fig. 1).

For $i=1$, we take all the patches $P^{\prime}$ in $\mathcal{T}_{1}$ satisfying the following two conditions:

$$
\begin{aligned}
& P^{\prime}=\mathcal{T}_{1}\left[B_{R+s_{0}+\eta_{1}+\eta_{2}+2 \varepsilon}(v)\right] \text { for some } v \in \mathbb{R}^{d}, \\
& P_{1}=\mathcal{T}_{1}\left[B_{R+s_{0}+\eta_{2}}(v)\right] .
\end{aligned}
$$

Because $\mathcal{T}_{1}$ satisfies the FPC, there exists a finite number $k_{1}$ of different patches satisfying the previous condition. We call these patches $P_{1,1}, \ldots, P_{1, k_{1}}$. Moreover, $k_{1}$ is bounded by $K$. Indeed, if $v$ and $v^{\prime}$ are two different points in $\mathbb{R}^{d}$ such that

$$
\begin{aligned}
P_{1, j} & =\mathcal{T}_{1}\left[B_{R+s_{0}+\eta_{1}+\eta_{2}+2 \varepsilon}(v)\right], \\
P_{1, i} & =\mathcal{T}_{1}\left[B_{R+s_{0}+\eta_{1}+\eta_{2}+2 \varepsilon}\left(v^{\prime}\right)\right],
\end{aligned}
$$


Fig. 1 Situation during the selection of the $P_{i, j}$ when $i \geq 2$

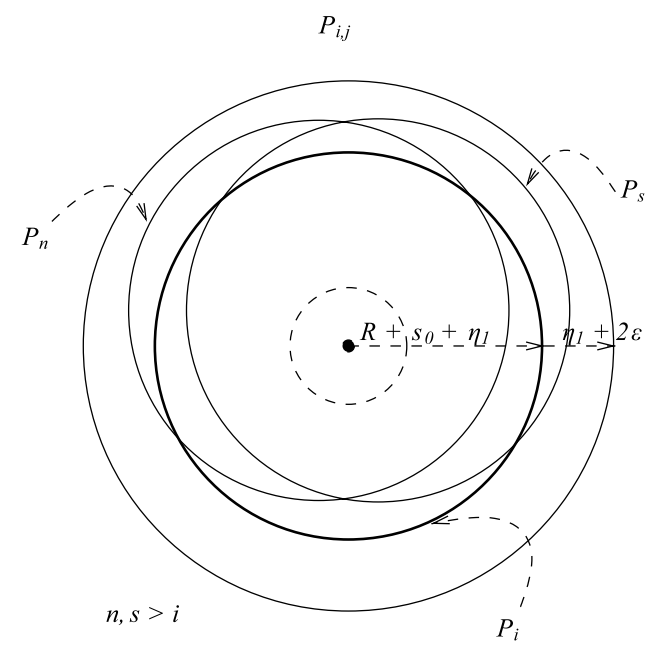

for some $1 \leq i, j \leq k_{1}$, then

$$
P_{1}=\mathcal{T}_{1}\left[B_{R+s_{0}+\eta_{2}}(v)\right]=\mathcal{T}_{1}\left[B_{R+s_{0}+\eta_{2}}\left(v^{\prime}\right)\right] .
$$

By Lemma 5, this implies that

$$
\left\|v-v^{\prime}\right\|>\frac{R+s_{0}+\eta_{2}}{M}
$$

It follows that in a ball of radius $\frac{R+s_{0}+\eta_{2}}{2 M}$ there is at most one point $w$ which is the center of some $P_{1, j}$. Since $\mathcal{T}_{1}$ is linearly recurrent with constant $L$ and for every $1 \leq j \leq k_{1}$,

$$
\operatorname{diam}\left(P_{1, j}\right) \leq 2\left(R+s_{0}+\eta_{1}+\eta_{2}+2 \varepsilon\right)+2 \eta_{1},
$$

all the patches $P_{1, j}$ appear in the ball $B_{2 L\left(R+s_{0}+2 \eta_{1}+\eta_{2}+2 \varepsilon\right)}(0)$ in $\mathcal{T}_{1}$. Using (3.5), this implies

$$
k_{1} \leq \frac{\operatorname{vol}\left(B_{2 L\left(R+s_{0}+2 \eta_{1}+\eta_{2}+2 \varepsilon\right)}(0)\right)}{\operatorname{vol}\left(B_{\frac{R+s_{0}+\eta_{2}}{2 M}}(0)\right)} \leq(8 L M)^{d} \leq K .
$$

For $1<i \leq l$, we take all the patches $P^{\prime}$ in $\mathcal{T}_{1}$ satisfying the following three conditions:

$$
\begin{aligned}
P^{\prime} & =\mathcal{T}_{1}\left[B_{R+s_{0}+\eta_{1}+\eta_{2}+2 \varepsilon}(v)\right] \quad \text { for some } v \in \mathbb{R}^{d}, \\
P_{i} & =\mathcal{T}_{1}\left[B_{R+s_{0}+\eta_{2}}(v)\right], \quad \text { if } \\
\mathcal{T}_{1} & {\left[B_{R+s_{0}+\eta_{2}}(v+t)\right]=P_{j} \quad \text { for some } t \in B_{2 \varepsilon}(0), \text { then } j \geq i . }
\end{aligned}
$$

As for the case $i=1$, we remark there is a finite number $k_{i}$ of different patches satisfying the previous conditions and that $k_{i}$ is smaller than $K$. We call these patches $P_{i, 1}, \ldots, P_{i, k_{i}}$. 
Remark 10 The linear recurrence of $\mathcal{T}_{1}$ and (3.5) imply that if $v \in \mathbb{R}^{d}$ satisfies

$$
\mathcal{T}_{1}\left[B_{R+s_{0}+\eta_{1}+\eta_{2}+2 \varepsilon}(v)\right]=P_{i, j}
$$

for some $1 \leq i \leq l$ and $1 \leq j \leq k_{i}$, then $\mathcal{T}_{1}\left[B_{R+s_{0}+\eta_{2}}(v+t)\right] \neq P_{i}$ for every $t \in$ $B_{2 \varepsilon}(0) \backslash\{0\}$.

Remark 11 By Remark 10 and (3.10), if $v \in \mathbb{R}^{d}$ satisfies

$$
\mathcal{T}_{1}\left[B_{R+s_{0}+\eta_{1}+\eta_{2}+2 \varepsilon}(v)\right]=P_{i, j}
$$

for some $1 \leq i \leq l$ and $1 \leq j \leq k_{i}$, then $\mathcal{T}_{1}\left[B_{R+s_{0}+\eta_{2}}(v+t)\right] \neq P_{s}$ for every $1 \leq s \leq i$ and $t \in B_{2 \varepsilon}(0) \backslash\{0\}$.

Remark 12 By the construction of the patches $P_{i, j}$, if $v \in \mathbb{R}^{d}$ satisfies

$$
\mathcal{T}_{1}\left[B_{R+s_{0}+\eta_{2}}(v)\right]=P_{i}
$$

for some $1 \leq i \leq l$ and $j>i$ whenever $\mathcal{T}_{1}\left[B_{R+s_{0}+\eta_{2}}(v+t)\right]=P_{j}$ for some $t \in$ $B_{2 \varepsilon}(0) \backslash\{0\}$, then

$$
\mathcal{T}_{1}\left[B_{R+s_{0}+\eta_{1}+\eta_{2}+2 \varepsilon}(v)\right]=P_{i, k}
$$

for some $1 \leq k \leq k_{i}$.

In the sequel we will show that

$$
\operatorname{freq}(P)=\sum_{i=1}^{l} \sum_{j=1}^{k_{i}} \operatorname{freq}\left(P_{i, j}\right)
$$

Lemma 13 Let $v \in \mathbb{R}^{d}$ be such that

$$
\mathcal{T}_{1}\left[B_{R+s_{0}+\eta_{1}+\eta_{2}+2 \varepsilon}(v)\right]=P_{i, j}
$$

for some $1 \leq i \leq l$ and $1 \leq j \leq k_{i}$. Then there exists a point $w(v) \in B_{\epsilon}(v)$ verifying $\mathcal{T}_{2}\left[B_{R}(w(v))\right]=P$ Moreover, if $v^{\prime} \neq v$, then $w\left(v^{\prime}\right) \neq w(v)$ and

$$
\sum_{i=1}^{l} \sum_{j=1}^{k_{i}} \operatorname{freq}\left(P_{i, j}\right) \leq \operatorname{freq}(P) .
$$

Proof Consider $v \in \mathbb{R}^{d}$ such that

$$
\mathcal{T}_{1}\left[B_{R+s_{0}+\eta_{1}+\eta_{2}+2 \varepsilon}(v)\right]=P_{i, j}
$$

for some $1 \leq i \leq l$ and $1 \leq j \leq k_{i}$. Since $\mathcal{T}_{1}\left[B_{R+s_{0}+\eta_{2}}(v)\right]=P_{i}$, we have

$$
\left(\mathcal{T}_{1}+v\right) \cap B_{R+s_{0}+\eta_{2}}(0)=\left(\mathcal{T}_{1}+v_{i}\right) \cap B_{R+s_{0}+\eta_{2}}(0) .
$$


Thus from Lemma 2 we obtain that there exists $t \in B_{\varepsilon}(0)$ verifying

$$
\left(\mathcal{T}_{2}+v+t\right) \cap B_{R+\eta_{2}}(0)=\left(\mathcal{T}_{2}+v_{i}\right) \cap B_{R+\eta_{2}}(0),
$$

which implies that $\mathcal{T}_{2}\left[B_{R}(v+t)\right]=P$. Now, if $v^{\prime} \in \mathbb{R}^{d}$ is another point such that

$$
\mathcal{T}_{1}\left[B_{R+s_{0}+\eta_{1}+\eta_{2}+2 \varepsilon}\left(v^{\prime}\right)\right]=P_{i^{\prime}, j^{\prime}}
$$

for some $1 \leq i^{\prime} \leq l$ and $1 \leq j^{\prime} \leq k_{i}^{\prime}$, in a similar way we get that there exists $t^{\prime} \in$ $B_{\varepsilon}(0)$ satisfying $\mathcal{T}_{2}\left[B_{R}\left(v^{\prime}+t^{\prime}\right)\right]=P$. Suppose that $v+t=v^{\prime}+t^{\prime}$. This implies that $\left\|v-v^{\prime}\right\|<2 \varepsilon$, i.e., $v-v^{\prime} \in B_{2 \varepsilon}(0)$. But since

$$
\begin{aligned}
P_{i, j} & =\mathcal{T}_{1}\left[B_{R+s_{0}+\eta_{1}+\eta_{2}+2 \varepsilon}(v)\right], \\
P_{i} & =\mathcal{T}_{1}\left[B_{R+s_{0}+\eta_{2}}(v)\right], \\
P_{i^{\prime}} & =\mathcal{T}_{1}\left[B_{R+s_{0}+\eta_{2}}\left(v+\left(v^{\prime}-v\right)\right)\right],
\end{aligned}
$$

the condition (3.10) implies that $i^{\prime} \geq i$. In the same way we obtain that $i^{\prime} \leq i$, which implies $i=i^{\prime}$. Since $2 \varepsilon<\frac{R+s_{0}}{M}$, we get that $v^{\prime}-v=0$. Hence we deduce that is possible to associate to each $v$ in $\mathbb{R}^{d}$ which satisfies

$$
\mathcal{T}_{1}\left[B_{R+s_{0}+\eta_{1}+\eta_{2}+2 \varepsilon}(v)\right]=P_{i, j}
$$

for some $1 \leq i \leq l$ and $1 \leq j \leq k_{i}$, a point $w(v) \in \mathbb{R}^{d}$ verifying

$$
\mathcal{T}_{2}\left[B_{R}(w(v))\right]=P
$$

and such that $w(v) \neq w\left(v^{\prime}\right)$ if $v \neq v^{\prime}$. Thus we deduce that

$$
\sum_{i=1}^{l} \sum_{j=1}^{k_{i}} \operatorname{freq}\left(P_{i, j}\right) \leq \operatorname{freq}(P) .
$$

Lemma 14 Let $v \in \mathbb{R}^{d}$ be such that $\mathcal{T}_{2}\left[B_{R}(v)\right]=P$. Then there exists a point $p(v) \in$ $B_{(2 l+1) \epsilon}(v)$ verifying

$$
\mathcal{T}_{1}\left[B_{R+s_{0}+\eta_{1}+\eta_{2}+2 \varepsilon}(p(v))\right]=P_{i, j}
$$

for some $1 \leq i \leq l$ and $1 \leq j \leq k_{i}$. Moreover, if $v^{\prime} \neq v$, then $p\left(v^{\prime}\right) \neq p(v)$ and

$$
\sum_{i=1}^{l} \sum_{j=1}^{k_{i}} \operatorname{freq}\left(P_{i, j}\right) \geq \operatorname{freq}(P) .
$$

Proof Let $v \in \mathbb{R}^{d}$ be such that

$$
\mathcal{T}_{2}\left[B_{R}(v)\right]=P
$$


Fig. 2 Situation before Step 0
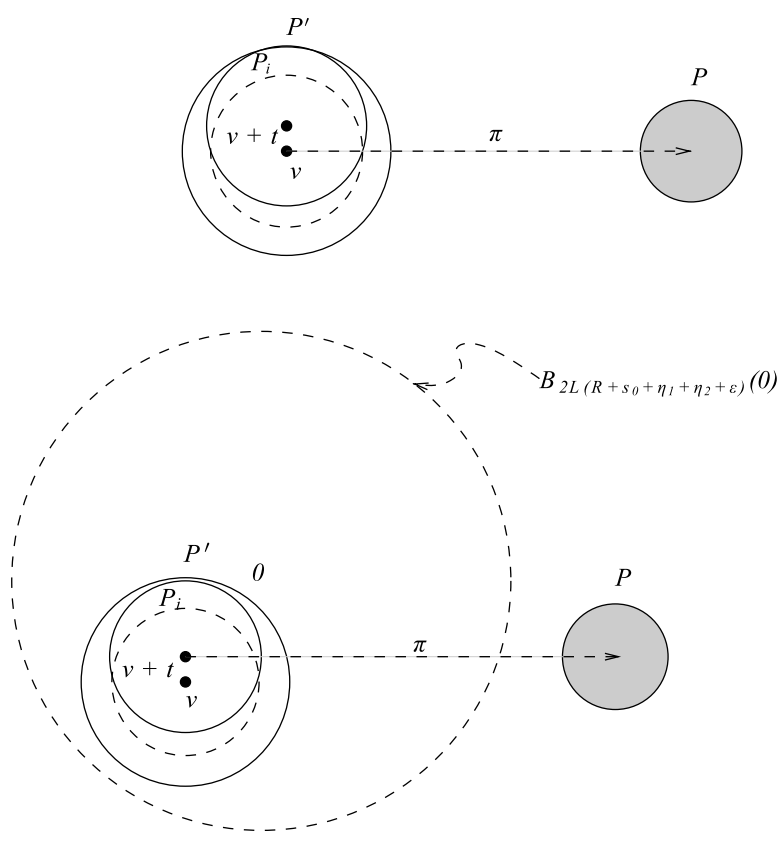

and consider

$$
P^{\prime}=\mathcal{T}_{1}\left[B_{R+s_{0}+\eta_{2}+\varepsilon}(v)\right] .
$$

Since $L$ is the constant of linear recurrence of $\mathcal{T}_{1}$ and

$$
\operatorname{diam}\left(P^{\prime}\right) \leq 2\left(R+s_{0}+\eta_{2}+\varepsilon\right)+2 \eta_{1},
$$

there exists a translate of $P^{\prime}$ the support of which is included in the ball

$$
B_{2 L\left(R+s_{0}+\eta_{1}+\eta_{2}+\varepsilon\right)}(0) \text {. }
$$

In other words, there exists $v^{\prime} \in B_{2 L}\left(R+s_{0}+\eta_{1}+\eta_{2}+\varepsilon\right)(0)$ such that the support of the patch $\mathcal{T}_{1} \llbracket B_{R+s_{0}+\eta_{2}+\varepsilon}\left(v^{\prime}\right) \rrbracket$ is contained in the ball $B_{2 L\left(R+s_{0}+\eta_{1}+\eta_{2}+\varepsilon\right)}(0)$ and satisfies

$$
\begin{aligned}
P^{\prime} & =\mathcal{T}_{1}\left[B_{R+s_{0}+\eta_{2}+\varepsilon}\left(v^{\prime}\right)\right] \\
& =\mathcal{T}_{1}\left[B_{R+s_{0}+\eta_{2}+\varepsilon}(v)\right] .
\end{aligned}
$$

This implies that

$$
\left(\mathcal{T}_{1}+v\right) \cap B_{R+s_{0}+\eta_{2}}(0)=\left(\mathcal{T}_{1}+v^{\prime}\right) \cap B_{R+s_{0}+\eta_{2}}(0) .
$$

So, by Lemma 2 there exists $t \in B_{\varepsilon}(0)$ verifying

$$
\left(\mathcal{T}_{2}+v^{\prime}+t\right) \cap B_{R+\eta_{2}}(0)=\left(\mathcal{T}_{2}+v\right) \cap B_{R+\eta_{2}}(0)
$$


Fig. 3 Situation when the algorithm finished

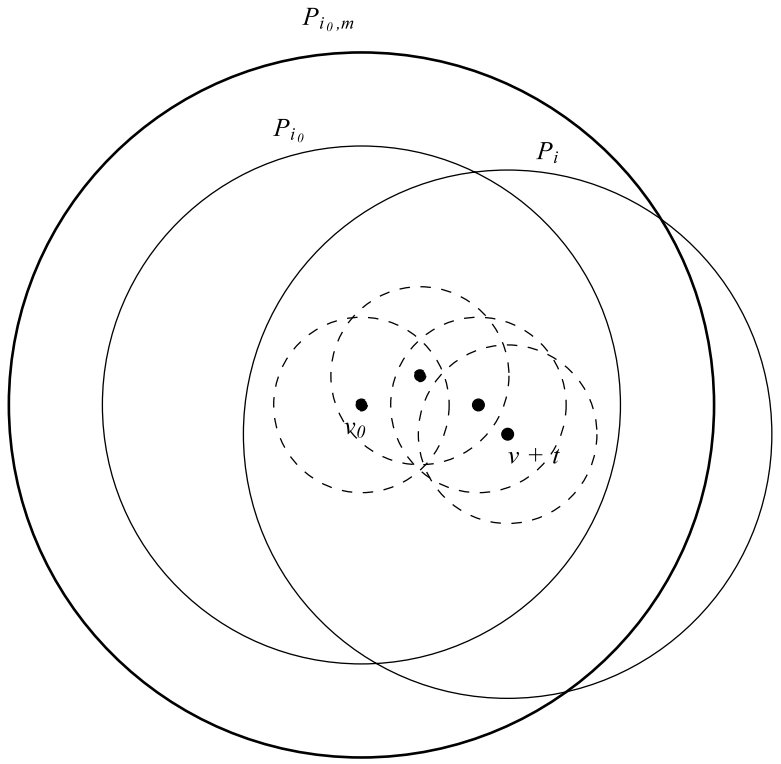

It follows that $\mathcal{T}_{2}\left[B_{R}\left(v^{\prime}+t\right)\right]=P$, and, since $v^{\prime}+t$ is in $B_{L\left(R+s_{0}+\eta_{1}+\eta_{2}+\varepsilon\right)}(0)$, we deduce that $v^{\prime}+t=v_{i}$ for some $1 \leq i \leq l$. Because $\mathcal{T}_{1}\left[B_{R+s_{0}+\eta_{2}}\left(v^{\prime}+t\right)\right]=P_{i}$ is included in $\mathcal{T}_{1}\left[B_{R+s_{0}+\eta_{2}+\varepsilon}\left(v^{\prime}\right)\right]=P^{\prime}$, we obtain that

$$
\mathcal{T}_{1}\left[B_{R+s_{0}+\eta_{2}}(v+t)\right]=P_{i} .
$$

Now, we will show that in the ball $B_{(2 l+1) \varepsilon}(v)$ there is a point $p(v)$ such that

$$
\mathcal{T}_{1}\left[B_{R+s_{0}+\eta_{1}+\eta_{2}+2 \varepsilon}(p(v))\right]=P_{m, j}
$$

for some $1 \leq m \leq l$ and $1 \leq j \leq k_{m}$. For that, consider the following algorithm (see Fig. 3):

Step 0: We put $v_{0}=v+t$ and $i_{0}=i$.

Step 1: We have $\mathcal{T}_{1}\left[B_{R+s_{0}+\eta_{2}}\left(v_{0}\right)\right]=P_{i_{0}}$.

If $\mathcal{T}_{1}\left[B_{R+s_{0}+\eta_{2}}\left(v_{0}+s\right)\right]=P_{j}$ for some $s \in B_{2 \varepsilon}(0)$ implies $j \geq i_{0}$, then from the definition of the patches $P_{i, k}$ we obtain that

$$
\mathcal{T}_{1}\left[B_{R+s_{0}+\eta_{1}+\eta_{2}+2 \varepsilon}\left(v_{0}\right)\right]=P_{i_{0}, m}
$$

for some $m$ in $\left\{1, \ldots, k_{i_{0}}\right\}$.

Step 2: If there exists $s \in B_{2 \varepsilon}(0)$ such that $\mathcal{T}_{1}\left[B_{R+s_{0}+\eta_{2}}\left(v_{0}+s\right)\right]=P_{j}$ with $j<i_{0}$, then we put

$$
i_{0}=\min \left\{j: \exists s \in B_{2 \varepsilon}(0) \text { such that } \mathcal{T}_{1}\left[B_{R+s_{0}+\eta_{2}}\left(v_{0}+s\right)\right]=P_{j}\right\} .
$$

If $s \in B_{2 \varepsilon}(0)$ is such that $\mathcal{T}_{1}\left[B_{R+s_{0}+\eta_{2}}\left(v_{0}+s\right)\right]=P_{i_{0}}$, then we put $v_{0}=v_{0}+s$. With these new values of $v_{0}$ and $i_{0}$, we go to Step 1 . 
This algorithm finishes in at most $l$ steps. The result is a point $p(v)=v_{0}$ the distance of which to $v$ is at most $(2 l+1) \varepsilon$ and such that

$$
\mathcal{T}_{1}\left[B_{R+s_{0}+\eta_{1}+\eta_{2}+2 \varepsilon}\left(v_{0}\right)\right]=P_{i_{0}, m}
$$

for some $m$ in $\left\{1, \ldots, k_{i_{0}}\right\}$.

If $w \in \mathbb{R}^{d}$ is another point satisfying $\mathcal{T}_{2}\left[B_{R}(w)\right]=P$, we have

$$
\begin{aligned}
\frac{R}{N} & \leq\|v-w\| \\
& \leq\|p(v)-v\|+\|p(v)-p(w)\|+\|p(w)-w\| \\
& \leq 2(2 l+1) \varepsilon+\|p(v)-p(w)\| .
\end{aligned}
$$

Thus we get

$$
0<\frac{R}{2 N}<\frac{R}{N}-2(2 l+1) \varepsilon \leq\|p(v)-p(w)\| .
$$

This implies that it is possible to associate to each $v$ in $\mathbb{R}^{d}$ which satisfies $\mathcal{T}_{2}\left[B_{R}(v)\right]=P$ a point $p(v) \in \mathbb{R}^{d}$ verifying

$$
\mathcal{T}_{1}\left[B_{R+s_{0}+\eta_{1}+\eta_{2}+2 \varepsilon}(p(v))\right]=P_{i, j}
$$

for some $1 \leq i \leq l$ and $1 \leq j \leq k_{i}$ and such that $p(v) \neq p(w)$ if $v \neq w$. Hence we deduce that

$$
\operatorname{freq}(P) \leq \sum_{i=1}^{l} \sum_{j=1}^{k_{i}} \operatorname{freq}\left(P_{i, j}\right) .
$$

From (3.11) and (3.12) we get

$$
\operatorname{freq}(P)=\sum_{i=1}^{l} \sum_{j=1}^{k_{i}} \operatorname{freq}\left(P_{i, j}\right) .
$$

As $R>\eta / 2$, there exists $k>0$ such that

$$
\eta \lambda^{k-2} \leq 2\left(R+s_{0}+\eta_{1}+\eta_{2}+2 \varepsilon\right)<\eta \lambda^{k-1} .
$$

Since

$$
2\left(R+s_{0}+\eta_{1}+\eta_{2}+2 \varepsilon\right) \leq \operatorname{diam}\left(P_{i, j}\right) \leq 2\left(R+s_{0}+\eta_{1}+\eta_{2}+2 \varepsilon\right)+2 \eta_{1}
$$

and $R \geq \eta \lambda^{\left\lceil\log _{\lambda} \frac{2 \eta_{1}}{\eta(\lambda-1)}\right\rceil}$, we have

$$
\eta \lambda^{k-2} \leq \operatorname{diam}\left(P_{i, j}\right)<\eta \lambda^{k} .
$$

Hence, by Proposition 7, we get

$$
\operatorname{freq}\left(P_{i, j}\right) \in\left\{\frac{f}{\lambda^{d k}}, \frac{f}{\lambda^{d(k-1)}}: f \in F\right\},
$$


where $F$ is the finite set of Proposition 7. Thus we obtain

$$
\operatorname{freq}(P)=\frac{f}{\lambda^{d k}},
$$

where $f$ is an element in

$$
F^{\prime}=\left\{\sum_{i=1}^{K} f_{i}: f_{i} \in F \cup \lambda^{d} F, \forall 1 \leq i \leq K\right\},
$$

which is a finite subset of $\mathbb{R}^{d}$.

Notice that

$$
2 R \leq \operatorname{diam}(P) \leq 2\left(R+\eta_{2}\right) .
$$

Thus from (3.14) we have

$$
\eta \lambda^{k-2}-2\left(s_{0}+\eta_{1}+\eta_{2}+2 \varepsilon\right) \leq \operatorname{diam}(P)<\eta \lambda^{k-1},
$$

and by the choice of $R$ in (3.5), we obtain

$$
\eta \lambda^{k-3} \leq \operatorname{diam}(P)<\eta \lambda^{k-1} .
$$

\section{Proof of Theorem 1}

By Proposition 9, there exist two finite sets $F_{1}$ and $F_{2}$ such that for $R>0$ and $P=$ $\mathcal{T}\left[B_{R}(0)\right]$, there exist $k_{1}$ and $k_{2}$ such that

$$
\operatorname{freq}(P)=\frac{f_{1}}{\lambda_{1}^{k_{1}}}=\frac{f_{2}}{\lambda_{2}^{k_{2}}}
$$

for some $f_{1} \in F_{1}$ and $f_{2} \in F_{2}$.

Because $F_{1}$ and $F_{2}$ are finite, we can find $a \in F_{1}, b \in F_{2}, n_{2}>n_{1}, m_{2}>m_{1}$ and patches $P_{1}$ and $P_{2}$ in $\mathcal{T}$ such that

$$
\begin{aligned}
& \operatorname{freq}\left(P_{1}\right)=\frac{a}{\lambda_{1}^{n_{1}}}=\frac{b}{\lambda_{2}^{m_{1}}}, \\
& \operatorname{freq}\left(P_{2}\right)=\frac{a}{\lambda_{1}^{n_{2}}}=\frac{b}{\lambda_{2}^{m_{2}}} .
\end{aligned}
$$

This implies that

$$
\lambda_{1}^{n_{2}-n_{1}}=\lambda_{2}^{m_{2}-m_{1}},
$$

which means that $\lambda_{1}$ and $\lambda_{2}$ are multiplicatively dependent.

Acknowledgements The authors acknowledge financial support from Nucleus Millennium PF04069-F, Ecos-Conicyt program C03E03, Fondecyt de Iniciación 11060002 and thank the Laboratoire Amiénois de Mathématiques Fondamentales et Appliquées of Université de Picardie Jules Verne, Centro de Modelamiento Matemático and Departamento de Ingeniería Matemática of Universidad de Chile, where part of this work has been done. 


\section{References}

1. Bès, A.: An extension of the Cobham-Semënov theorem. J. Symb. Log. 65, 201-211 (2000)

2. Bruyère, V., Hansel, G.: In: Recognizable Sets of Numbers in Nonstandard Bases. Lecture Notes in Comput. Sci., vol. 911, pp. 167-179. Springer, Berlin (1995)

3. Bruyère, V., Hansel, G.: Bertrand numeration systems and recognizability. Theor. Comput. Sci. 181, 17-43 (1997). Latin American Theoretical Informatics (Valparaíso, 1995)

4. Bruyère, V., Hansel, G., Michaux, C., Villemaire, R.: Logic and p-recognizable sets of integers. Bull. Belg. Math. Soc. S. Stevin 1, 191-238 (1994)

5. Cobham, A.: On the base-dependence of sets of numbers recognizable by finite automata. Math. Syst. Theory 3, 186-192 (1969)

6. Cobham, A.: Uniform tag sequences. Math. Syst. Theory 6, 164-192 (1972)

7. Durand, F.: Sur les ensembles d'entiers reconnaissables. J. Théor. Nombres Bordeaux 10, 65-84 (1998)

8. Durand, F.: A generalization of Cobham's theorem. Theory Comput. Syst. 31, 169-185 (1998)

9. Durand, F.: A theorem of Cobham for non-primitive substitutions. Acta Arith. 104, 225-241 (2002)

10. Eilenberg, S.: Automata, Languages, and Machines, vol. A. Pure and Applied Mathematics, vol. 58. Academic Press, New York (1974)

11. Fabre, S.: Une généralisation du théorème de Cobham. Acta Arith. 67, 197-208 (1994)

12. Fagnot, I.: Sur les facteurs des mots automatiques. Theor. Comput. Sci. 172, 67-89 (1997)

13. Hansel, G.: A propos d'un théorème de Cobham. In: Perrin, D. (ed.) Actes de la Fête des Mots. GRECO de Programmation, Rouen (1982)

14. Hansel, G.: Systèmes de numération indépendants et syndéticité. Theor. Comput. Sci. 204, 119-130 (1998)

15. Holton, C., Zamboni, L.: Directed graphs and substitutions. Theor. Comput. Syst. 34, 545-564 (2001)

16. Holton, C., Radin, C., Sadun, L.: Conjugacies for tiling dynamical systems. Commun. Math. Phys. 254(2), 343-359 (2005)

17. Michaux, C., Villemaire, R.: Presburger arithmetic and recognizability of sets of natural numbers by automata: new proofs of Cobham's theorem and Semenov's theorem. Ann. Pure Appl. Log. 77, 251-277 (1996)

18. Petersen, K.: Factor maps between tiling dynamical systems. Forum Math. 11(4), 503-512 (1999)

19. Radin, C., Sadun, L.: Isomorphism of hierarchical structures. Ergod. Theor. Dyn. Syst. 21(4), 12391248 (2001)

20. Rudolph, D.J.: Markov tilings of $R^{n}$ and representations of $R^{n}$ actions. In: Measure and Measurable Dynamics, Rochester, NY, 1987. Contemp. Math., vol. 94, pp. 271-290. Amer. Math. Soc., Providence (1989)

21. Semenov, A.L.: The Presburger nature of predicates that are regular in two number systems. Sib. Math. J. 18, 289-299 (1977)

22. Solomyak, B.: Dynamics of self-similar tilings. Ergod. Theory Dyn. Syst. 17, 695-738 (1997)

23. Solomyak, B.: Nonperiodicity implies unique composition for self-similar translationally finite tilings. Discrete Comput. Geom. 20, 265-279 (1998) 